\title{
Associations of Periodontal Damage and Tooth Loss with Atherogenic Factors among Patients with Type 2 Diabetes Mellitus
}

\author{
Tae Furukawa ${ }^{1}$, Kenji Wakai ${ }^{2}$, Kunio Yamanouchi ${ }^{3}$, Yoshiharu Oshida ${ }^{1}$, Masaru Miyao $^{4}$, \\ Tomoyuki Watanabe ${ }^{5}$ and Yuzo Sato ${ }^{6}$
}

\begin{abstract}
Objective To clarify the associations of periodontal damage and tooth loss with atherogenic factors among diabetic patients.

Methods We examined the correlations of age, sex, smoking, oral hygiene score, blood pressure, body mass index, and blood chemical data with the mean depth of periodontal pockets or the number of remaining teeth.

Patients One hundred outpatients with type 2 diabetes aged 29 to 77 years.

Results The mean depth of periodontal pockets was significantly associated with smoking, oral hygiene score, and HbA1c; the Spearman correlation coefficients (r) were 0.220, 0.417, and 0.260, respectively. Age, oral hygiene score, and $\mathrm{HbAlc}$ were inversely correlated with the number of remaining teeth $(\mathrm{r}=-0.306$, -0.287 , and -0.275 , respectively). Serum total cholesterol was significantly correlated with the mean depth of pockets after adjustment for smoking, oral hygiene score, and HbAlc $(r=0.211 ; \mathrm{P}=0.044)$, while serum HDL cholesterol tended to be negatively associated with depth $(\mathrm{r}=-0.202 ; \mathrm{P}=0.055)$. Serum HDL cholesterol was also associated with an increased number of teeth, which remained significant after adjustment for age, oral hygiene score, and $\mathrm{HbA} 1 \mathrm{c}(\mathrm{r}=0.202 ; \mathrm{P}=0.048)$. The estimated glomerular filtration rate was significantly and positively correlated with the number of teeth in the univariate analysis, although consideration of the potential confounding factors somewhat weakened the association ( $\mathrm{r}=0.186 ; \mathrm{P}=0.069)$.

Conclusions We may expect better management of oral health in diabetic patients with control of dyslipidemia in addition to blood glucose.
\end{abstract}

Key words: diabetic patients, periodontal disease, hyperlipidemia, dyslipidemia, atherosclerosis

(DOI: 10.2169/internalmedicine.46.0106)

\section{Introduction}

Periodontitis has been referred to as the sixth complication of diabetes after microangiopathy including retinopathy, nephropathy, neuropathy, macrovascular disease, and delayed wound healing $(1,2)$. Many studies have long recognized that the disease is common among diabetic patients and becomes worse with the progression of diabetes. Type 2 diabetic patients are more likely to have deep periodontal pockets and to lose teeth than non-diabetic controls (3). Deterioration of periodontal condition and subsequent tooth loss result in impaired chewing ability and unhealthier diets such as decreased intake of fruits and vegetables (4). In addition, chewing ability may be an independent predictor of physical fitness measurements in the elderly (5). Thus, pre-

${ }^{1}$ Research Center of Health, Physical Fitness and Sports, Nagoya University, Nagoya, ${ }^{2}$ Department of Preventive Medicine/Biostatistics and Medical Decision Making, Nagoya University Graduate School of Medicine, Nagoya, ${ }^{3}$ Yamanouchi Clinical Preventive Research Office of Diabetes Mellitus, Nagakute, ${ }^{4}$ Information Technology Center, Nagoya University, Nagoya, ${ }^{5}$ Clinical Research Center, National Hospital Organization Nagoya Medical Center, Nagoya and ${ }^{6}$ Department of Health Science, Faculty of Psychological \& Physical Science, Aichi Gakuin University, Nisshin

Received for publication February 16, 2007; Accepted for publication May 2, 2007

Correspondence to Dr. Yuzo Sato, satoy@dpc.agu.ac.jp 
ventive dental care aimed to maintain chewing ability may enhance activities of daily living (ADL) and quality of life in diabetic patients.

Although the pathogenic role of a microbial plaque and hyperglycemia is beyond doubt, several other factors could influence the initiation and progression of periodontal damage in diabetics. Dyslipidemia combined with hyperglycemia may be responsible for deregulation of cytokine production by immune cells and increased inflammatory destruction of periodontal tissue (2). This is supported by recent investigations, which found associations between impaired lipid metabolism and periodontal disease (6-10). Furthermore, atherosclerotic diseases such as cardiovascular diseases have recently been associated with periodontal disease $(11,12)$. Atherogenic factors other than hyperglycemia including hypertension, dyslipidemia, and obesity may induce atherosclerosis and subsequent ischemia also in periodontal tissue. Chronic kidney disease has also attracted attention in relation to periodontal disease (13).

Whether atherogenic factors other than hyperglycemia are linked with periodontal damage, however, is still being debated, and previous studies have reported inconsistent results (6-10, 13-21). Further, only a few studies examined such associations among patients with diabetes (14). The associations may differ between diabetics and non-diabetics. Thus, we investigated the associations of blood pressure, body mass index (BMI), and blood chemical data relating atherosclerosis with oral health in patients with diabetes, using the mean depth of periodontal pockets and the number of remaining teeth as indices.

\section{Patients and Methods}

\section{Patients}

The subjects of the present study were 100 outpatients with type 2 diabetes mellitus at the Aichi Medical University Hospital (from March to October 2001) and the Fushimi Clinic (Nagoya, Japan; from November 2002 to January 2003). They were enrolled from diabetic outpatient clinics when one of the authors (TF: a dentist) was available. They included 72 men and 28 women aged 29 to 77 years. Mean ages ( \pm standard deviation) of the patients were $59 \pm 7$ years for men and $60 \pm 11$ years for women. The diagnosis of diabetes was made according to the criteria of the Japan Diabetes Society (22). The Ethical Board of the Research Center of Health, Physical Fitness and Sports, Nagoya University approved the protocol of this investigation, and all participants verbally provided informed consent.

\section{General health examination}

The patients underwent a routine check-up including anthropometric and manometric measurements and blood chemical analysis. Blood pressure was measured using a mercury sphygmomanometer, with patients in a sitting position. Because blood was not necessarily drawn when the pa- tients were fasting, we did not adopt data on serum triglyceride. The blood samples were analyzed for HbA1c and serum total cholesterol, high-density lipoprotein (HDL) cholesterol, and creatinine. Serum creatinine was determined using the enzymatic method. The method and period of treatment were abstracted from the clinical charts. Diabetic retinopathy was examined at least annually by expert ophthalmologists using ophthalmoscopy. Urinalysis was performed to check albuminuria and/or proteinuria. Nephropathy was diagnosed in patients with urine microalbumin levels of greater than $30 \mathrm{mg} / \mathrm{dl}$ and/or with positive dip stick (23).

Information on smoking habits was obtained using a selfadministered questionnaire, which elicited the number of cigarettes smoked per day in six categories: 0, 1-9, 10-19, 20-29, 30-39, or $\geq 40$ cigarettes/day.

\section{Dental examination}

Subjects underwent a series of dental examinations including the number of decayed, missing, and filled teeth. Twenty-eight was the highest number of remaining teeth because we excluded third molars. In addition, to assess periodontal condition, we probed all existing teeth to determine depth of periodontal pockets with a specially-designed periodontal probe. The pocket depth was averaged over all teeth of each subject. The depth was not available for those without remaining teeth. The oral hygiene status was scored as follows: 0: almost no plaque; 1: plaque covers less than one third of tooth surface; and 2: plaque covers more than one third of tooth surface. One of the authors (TF) conducted all dental examinations.

\section{Statistical analysis}

The associations between potential demographic (age and sex) or conventional [smoking (number of cigarettes smoked per day), oral hygiene score, HbAlc, and duration of therapy for diabetes] risk factors for periodontal disease (24) and the mean depth of periodontal pockets or number of remaining teeth were examined by calculating Spearman correlation coefficients. These coefficients were also computed for medical factors including blood pressure, serum total cholesterol, BMI, and estimated glomerular filtration rate (GFR). GFR was estimated using the MDRD (Modification of Diet in Renal Disease Study Group) 4-variable formula (25): Estimated GFR $\left(\mathrm{ml} / \mathrm{min} / 1.73 \mathrm{~m}^{2}\right)=175 \times($ serum creatinine $[\mathrm{mg} / \mathrm{dl}])^{-1.154} \times(\text { age }[\text { years }])^{-0.203} \times 0.742$ (if woman). We further adjusted the correlation coefficients of medical variables for the demographic or conventional risk factors significantly correlated with the mean depth of periodontal pockets or number of teeth, by computing partial correlation coefficients. The factors were smoking, oral hygiene score, and $\mathrm{HbA} 1 \mathrm{c}$ for the mean depth of pockets, and age, oral hygiene score, and HbA1c for number of teeth. In addition, we repeated these analyses after excluding 33 current smokers or 34 individuals without 20 teeth. We also carried out a similar analysis for oral hygiene score. All $\mathrm{P}$ values were two-sided, and all the analyses were performed using the 
Table 1. Characteristics of Study Subjects with Type 2 Diabetes Mellitus $(\mathbf{n}=\mathbf{1 0 0})$

\begin{tabular}{lc}
\hline Age (years) & $59.1 \pm 8.4$ \\
Female & $28.0 \%$ \\
Duration of therapy for diabetes (years) & $12.6 \pm 7.2$ \\
HbA1c (\%) & $7.8 \pm 1.3$ \\
Diabetic nephropathy (yes) & $26.0 \%$ \\
Diabetic retinopathy (yes) $)^{\mathrm{a})}$ & $35.7 \%$ \\
Treatment) & \\
Diet and exercise only & $17.2 \%$ \\
With oral drugs & $48.5 \%$ \\
With insulin injection & $34.3 \%$ \\
Number of decayed teeth & $0.5 \pm 1.6$ \\
Number of filled teeth & $8.2 \pm 5.2$ \\
Systolic blood pressure $(\mathrm{mmHg})$ & $129.7 \pm 12.0$ \\
Diastolic blood pressure $(\mathrm{mmHg})$ & $77.1 \pm 7.7$ \\
Serum total cholesterol $(\mathrm{mg} / \mathrm{dl})$ & $200.9 \pm 30.1$ \\
Serum HDL cholesterol $(\mathrm{mg} / \mathrm{dl})$ & $52.1 \pm 12.9$ \\
Body mass index $\left(\mathrm{kg} / \mathrm{m}^{2}\right)^{\mathrm{a})}$ & $23.8 \pm 3.4$ \\
Serum creatinine $(\mathrm{mg} / \mathrm{dl})$ & $0.83 \pm 0.26$ \\
Estimated GFR $\left(\mathrm{ml} / \mathrm{min} / 1.73 \mathrm{~m}{ }^{2}\right)$ & $94.5 \pm 24.4$ \\
\hline
\end{tabular}

Plus-minus values are means $\pm \mathrm{SD}$.

HDL: high-density lipoprotein; GFR: glomerular filtration rate.

a) Data were missing for two subjects.

b) Data were missing for one subject.

Statistical Analysis System, version 9.1 (SAS Institute Inc., Cary, NC, USA) (26).

\section{Results}

Table 1 shows the selected characteristics of the 100 eligible subjects. They have been treated for diabetes for a mean of more than 10 years and included a substantial number of patients with nephropathy $(26.0 \%)$ and/or retinopathy $(35.7 \%)$. About one-third $(34.3 \%)$ of the patients were treated with insulin injection. The mean depth of periodontal pockets in more than $80 \%$ of subjects ranged from 2.0 to $4.4 \mathrm{~mm}$ with a peak of the distribution in $2.0-2.4 \mathrm{~mm}$ (Fig. 1). Twenty or more teeth were kept in two thirds $(66.0 \%)$ of the patients, and so were 25 or more teeth in one-third (36.0\%) (Fig. 2).

For age, sex, and conventional risk factors for periodontal disease, the mean depth of periodontal pockets was positively and significantly associated with smoking, oral hygiene score, and HbA1c; the Spearman correlation coefficients (r) were $0.220,0.417$, and 0.260 , respectively (Table 2). On the other hand, age, oral hygiene score, and HbA1c were inversely correlated with the number of remaining teeth ( $r=-0.306,-0.287$, and -0.275 , respectively).

Serum total cholesterol was significantly correlated with the mean depth of pockets after adjustment for smoking, oral hygiene score, and $\mathrm{HbA1c}(\mathrm{r}=0.211 ; \mathrm{P}=0.044)$, while the serum HDL cholesterol tended to be negatively associated with the depth $(\mathrm{r}=-0.202 ; \mathrm{P}=0.055)$ (Table 3$)$. Serum HDL cholesterol was also associated with an increased number of teeth, which remained significant after adjustment for

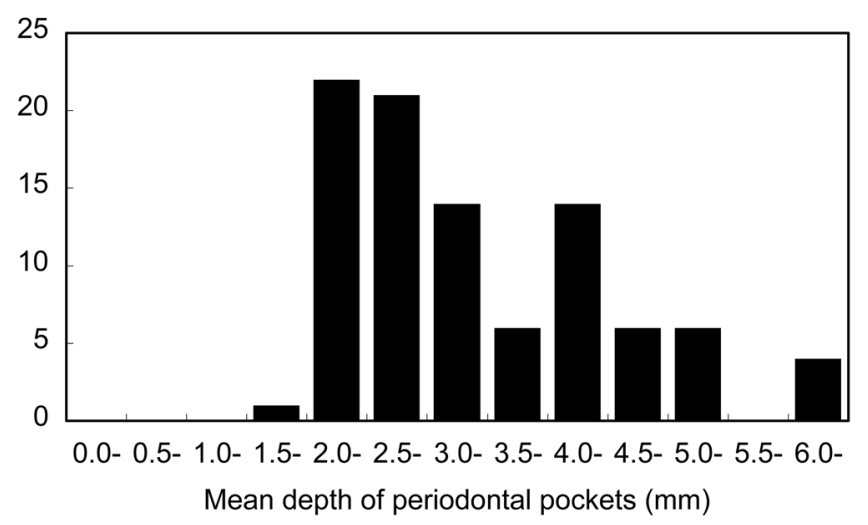

Figure 1. Distribution of study subjects by mean depth of periodontal pockets $(\mathrm{mm})$.

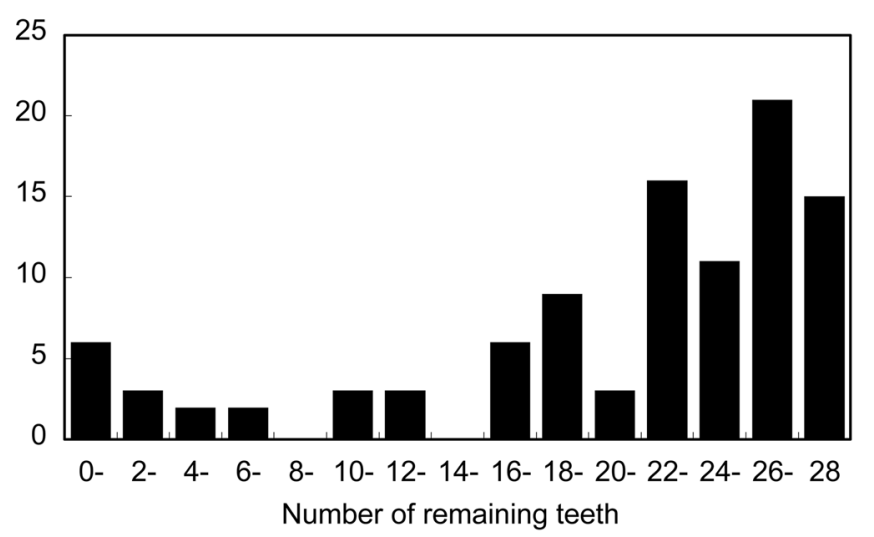

Figure 2. Distribution of study subjects by number of remaining teeth.

age, oral hygiene score, and HbA1c $(r=0.202 ; \mathrm{P}=0.048)$. The estimated GFR was significantly and positively correlated with the number of teeth in the univariate analysis, although consideration of the potential confounding factors somewhat weakened the association $(\mathrm{r}=0.186 ; \mathrm{P}=0.069)$. No clear association was observed between the mean depth of pockets or number of teeth and blood pressure or BMI.

The positive correlations between serum total cholesterol and the mean depth of periodontal pockets or serum HDL cholesterol and number of teeth and the negative correlation between serum HDL cholesterol and the mean pocket depth were still observed in the multivariate analyses limited to non-smokers or participants with 20 or more teeth (Table 4). Most of these associations, however, did not reach statistical significance probably due to the small sample size. In nonsmokers, estimated GFR was positively associated with the number of teeth after adjustment for age, oral hygiene score, and $\mathrm{HbA} 1 \mathrm{c}$.

We also conducted a similar analysis for oral hygiene score as those for the mean pocket depth and number of remaining teeth. Of the conventional risk factors for periodontal disease listed in Table 2, smoking and HbA1c were positively correlated with oral hygiene score $(\mathrm{r}=0.258 ; \mathrm{P}=0.010$ for smoking and $\mathrm{r}=0.196 ; \mathrm{P}=0.050$ for $\mathrm{HbA1c}$ ). Systolic and diastolic blood pressure, serum total and HDL cholesterol, BMI, and estimated GFR, however, were not associated with 
Table 2. Spearman Correlation Coefficients (r) between Age, Sex, or Conventional Risk Factors for Periodontal Disease and Mean Depth of Periodontal Pockets or Number of Teeth

\begin{tabular}{|c|c|c|c|c|}
\hline & \multicolumn{2}{|c|}{$\begin{array}{c}\text { Mean depth of } \\
\text { periodontal pockets } \\
(n=94)\end{array}$} & \multicolumn{2}{|c|}{$\begin{array}{l}\text { Number of teeth } \\
(n=100)\end{array}$} \\
\hline & $r$ & $\mathrm{P}$ & $r$ & $\mathrm{P}$ \\
\hline Age & 0.098 & 0.35 & -0.306 & 0.002 \\
\hline Female sex & -0.113 & 0.28 & -0.019 & 0.85 \\
\hline Smoking & 0.220 & 0.033 & 0.012 & 0.91 \\
\hline Oral hygiene score & 0.417 & $<0.001$ & -0.287 & 0.004 \\
\hline $\mathrm{HbA} 1 \mathrm{c}$ & 0.260 & 0.011 & -0.275 & 0.006 \\
\hline Duration of therapy for diabetes & 0.093 & 0.37 & -0.020 & 0.85 \\
\hline
\end{tabular}

Table 3. Spearman Correlation Coefficients (r) between Blood Pressure, Serum Cholesterol, Body Mass Index, or Estimated GFR and Mean Depth of Periodontal Pockets or Number of Teeth

\begin{tabular}{|c|c|c|c|c|c|c|c|c|}
\hline & \multicolumn{4}{|c|}{$\begin{array}{l}\text { Mean depth of periodontal } \\
\text { pockets } \\
(\mathrm{n}=94)\end{array}$} & \multicolumn{4}{|c|}{$\begin{array}{l}\text { Number of teeth } \\
\qquad(n=100)\end{array}$} \\
\hline & \multicolumn{2}{|c|}{ Crude } & \multicolumn{2}{|c|}{ Adjusted $^{\text {a) }}$} & \multicolumn{2}{|c|}{ Crude } & \multicolumn{2}{|c|}{ Adjusted $^{b)}$} \\
\hline & $r$ & $\mathrm{P}$ & $r$ & $\mathrm{P}$ & $r$ & $\mathrm{P}$ & $r$ & $\mathrm{P}$ \\
\hline Systolic blood pressure & -0.047 & 0.65 & -0.028 & 0.79 & -0.116 & 0.25 & -0.116 & 0.26 \\
\hline Diastolic blood pressure & -0.082 & 0.43 & -0.108 & 0.31 & -0.055 & 0.58 & -0.125 & 0.22 \\
\hline Serum total cholesterol & 0.182 & 0.078 & 0.211 & 0.044 & -0.029 & 0.78 & -0.057 & 0.58 \\
\hline Serum HDL cholesterol & -0.191 & 0.065 & -0.202 & 0.055 & 0.205 & 0.041 & 0.202 & 0.048 \\
\hline Body mass index & -0.042 & 0.69 & -0.071 & 0.51 & -0.069 & 0.50 & -0.090 & 0.38 \\
\hline Estimated GFR & -0.140 & 0.18 & -0.159 & 0.13 & 0.212 & 0.034 & 0.186 & 0.069 \\
\hline
\end{tabular}

HDL: high-density lipoprotein; GFR: glomerular filtration rate.

a) Adjusted for smoking, oral hygiene score, and $\mathrm{HbA} 1 \mathrm{c}$.

b) Adjusted for age, oral hygiene score, and HbA1c.

Table 4. Spearman Correlation Coefficients (r) between Blood Pressure, Serum Cholesterol, Body Mass Index, or Estimated GFR and Mean Depth of Periodontal Pockets or Number of Teeth among Non-smokers or Those with $>=20$ Teeth

\begin{tabular}{|c|c|c|c|c|c|c|c|c|c|c|c|c|c|c|c|c|}
\hline & \multicolumn{8}{|c|}{ Non-smokers } & \multicolumn{8}{|c|}{ Those with >= 20 teeth } \\
\hline & \multicolumn{4}{|c|}{$\begin{array}{l}\text { Mean depth of periodontal } \\
\text { pockets } \\
(\mathrm{n}=62)\end{array}$} & \multicolumn{4}{|c|}{$\begin{array}{l}\text { Number of teeth } \\
(n=67)\end{array}$} & \multicolumn{4}{|c|}{$\begin{array}{l}\text { Mean depth of periodontal } \\
\text { pockets } \\
(\mathrm{n}=65)\end{array}$} & \multicolumn{4}{|c|}{$\begin{array}{c}\text { Number of teeth } \\
(n=66)\end{array}$} \\
\hline & \multicolumn{2}{|c|}{ Crude } & \multicolumn{2}{|c|}{ Adjusted $^{a)}$} & \multicolumn{2}{|c|}{ Crude } & \multicolumn{2}{|c|}{ Adjusted $^{\text {b) }}$} & \multicolumn{2}{|c|}{ Crude } & \multicolumn{2}{|c|}{ Adjusted $^{()}$} & \multicolumn{2}{|c|}{ Crude } & \multicolumn{2}{|c|}{ Adjusted $^{\text {b) }}$} \\
\hline & $r$ & $\mathrm{P}$ & $r$ & $\mathrm{P}$ & $\mathrm{r}$ & $\mathrm{P}$ & $\mathrm{r}$ & $\mathrm{P}$ & $r$ & $\mathrm{P}$ & $r$ & $\mathrm{P}$ & $r$ & $\mathrm{P}$ & $r$ & $\mathrm{P}$ \\
\hline Systolic blood pressure & 0.039 & 0.76 & 0.026 & 0.84 & -0.192 & 0.12 & -0.143 & 0.26 & -0.079 & 0.53 & -0.058 & 0.66 & -0.139 & 0.27 & -0.187 & 0.14 \\
\hline Diastolic blood pressure & -0.166 & 0.20 & -0.158 & 0.23 & 0.031 & 0.80 & -0.013 & 0.92 & -0.105 & 0.40 & -0.096 & 0.46 & -0.037 & 0.77 & -0.095 & 0.46 \\
\hline Serum total cholesterol & 0.194 & 0.13 & 0.233 & 0.073 & 0.077 & 0.53 & 0.110 & 0.39 & 0.142 & 0.26 & 0.213 & 0.097 & 0.043 & 0.73 & -0.005 & 0.97 \\
\hline Serum HDL cholesterol & -0.216 & 0.091 & -0.203 & 0.12 & 0.191 & 0.12 & 0.179 & 0.16 & -0.282 & 0.023 & -0.250 & 0.050 & 0.364 & 0.003 & 0.336 & 0.007 \\
\hline Body mass index & -0.174 & 0.18 & -0.184 & 0.17 & -0.051 & 0.69 & -0.091 & 0.48 & 0.084 & 0.51 & 0.037 & 0.78 & -0.276 & 0.026 & -0.250 & 0.050 \\
\hline Estimated GFR & -0.142 & 0.27 & -0.135 & 0.30 & 0.329 & 0.007 & 0.309 & 0.013 & -0.101 & 0.43 & -0.083 & 0.52 & 0.102 & 0.41 & 0.071 & 0.58 \\
\hline
\end{tabular}

oral hygiene score both in univariate analysis and in multivariate analysis with adjustment for smoking and $\mathrm{HbA1c}$; all the absolute values of Spearman correlation coefficients were smaller than 0.1 with $\mathrm{P}$ values $>0.4$.

\section{Discussion}

The present study investigated possible associations of atherogenic factors and periodontal conditions or tooth loss in patients with type 2 diabetes. Hypercholesteremia was found to be correlated with the depth of periodontal pockets independently of hyperglycemia. On the contrary, serum HDL cholesterol was inversely associated with tooth loss and tended to be so with the pocket depth even after considering confounders. Renal function assessed by the estimated GFR was correlated with the number of remaining teeth, but the adjustment for potential confounding factors somewhat attenuated the association. 
Among atherogenic factors, the elevated serum total cholesterol was associated with periodontal damage assessed by the mean depth of periodontal pockets. Together with hyperglycemia, atherosclerosis will be accelerated by hypercholesteremia $(27,28)$, which may develop ischemia in periodontal tissue as tissue ischemia from infarcts in coronary heart disease and stroke. Diabetic microangiopathy contributes to compromised delivery of nutrients to surrounding tissues and poor elimination of metabolic waste products (29). Periodontal tissue damage may thus be more expected in diabetics with hypercholesteremia, as found in our study. Some (6, 9) but not all $(7,10,14-16,20)$ previous studies have associated hypercholesteremia with periodontal disease.

On the other hand, a high level of serum HDL cholesterol suppresses atherosclerosis $(27,28)$, and therefore may be related to a reduced risk of periodontal disease and tooth loss, which was also suggested in the present study. Some reports associated a high level of HDL cholesterol with a lower risk of periodontal disease $(7,10)$ whereas others found no such association $(8,14-16,20)$.

Periodontal disease and dyslipidemia may be more directly related (2). Elevated serum lipids including lowdensity lipoprotein cholesterol cause altered immune cell function resulting in impaired tissue response mainly due to increased production of proinflammatory cytokines such as TNF- $\alpha$ (tumor necrosis factor-alpha) and IL-1 $\beta$ (interleukin1 beta) by polymorphonuclear leukocytes and decreased production of essential polypeptide growth factors such as PDGF (platelet-derived growth factor), TGF- $\beta 1$ (transforming growth factor-beta 1), and bFGF (basic fibroblast growth factor) by tissue macrophages. Additionally, elevated serum lipids may cause a systemic monocytic hyperresponse trait leading to maintained elevations of serum TNF- $\alpha$ and IL-1 $\beta$. Compromised tissue response may predispose to periodontitis. On the other hand, periodontitis results in further elevation of serum lipid levels through systemic actions of proinflammatory cytokines (IL-1 $\beta /$ TNF- $\alpha$ ). Decreased serum HDL cholesterol levels were also reported in relation to infection to periodontal pathogens (30). Further studies that measure the inflammatory markers and evaluate the effect of treatment for hyperlipidemia on periodontal disease are warranted to support our findings.

Renal function evaluated using estimated GFR was correlated with number of teeth. Although the correlation was not statistically independent of age, oral hygiene score, and HbA1c, our study suggests that lowered filtration function of the kidneys due to diabetic nephropathy or other causes may have some deteriorating effect on teeth during longterm diabetes. Elevated serum creatinine has also been reported to be associated with advancing retinopathy and changes in vascular structure $(31,32)$. Similarly, decreased kidney function might indicate damage of periodontal tissue by changing the structure of arteries that nourish the tissue. In fact, Kshirsagar et al (13) recently reported an association of periodontal damage or tooth loss with decreased renal function, although previous investigation does not support their findings (14).

Hypertension has been reported to be associated with periodontal disease as latent risks for ischemic heart disease in several studies $(7,15,16,20,21)$, but in the present study no clear relation of the periodontal pockets or tooth loss was found with hypertension. It is possible, however, that the correlates of periodontal disease in patients with a long history of diabetes are different from those in the general population. Obesity has recently emerged as a possible risk indicator of periodontal disease $(10,17,18)$, although we and other investigators $(7,15,16,19)$ found no support for it. Obesity is closely associated with type 2 diabetes (33), but weight loss is one of the symptoms of the disease (34). On the other hand, weight gain is often observed after the initiation of therapy with sulphonylurea or insulin, which suggests a "catch-up" weight re-gain (34). The role of obesity in periodontal disease, therefore, might be obscured in cross-sectional studies among patients with diabetes. The present study showed that duration of therapy was not significantly correlated with the depth of periodontal pockets or tooth loss. Diabetic control may be more important than the duration of treatment to maintain a good periodontal condition. Sandberg et al (35) also did not show any correlation between the prevalence or the severity of periodontal disease and the duration of diabetes.

Some methodological issues warrant consideration. First, because this is a cross-sectional study, it is difficult to make causal inferences based merely on its findings. Prospective and/or interventional studies are required to confirm the association of dyslipidemia with periodontal disease. Second, we could not obtain a detailed past smoking history for the participants, which may have led to overlooking an association of smoking with tooth loss. Finally, some teeth may have been lost due to dental caries. This, however, would have apparently attenuated the correlation between serum HDL cholesterol and the number of remaining teeth because previous caries seem not to be related to HDL cholesterol.

In summary, in addition to hyperglycemia, hypercholesteremia was associated with deeper periodontal pockets, and so was the low level of serum HDL cholesterol with tooth loss. If our findings are further confirmed, we may expect better management of oral health in diabetic patients with improvements in not only plaque and blood glucose control but also in control of dyslipidemia.

We thank Dr. G. Bajotto for his suggestions regarding the English language.

\section{References}


2. Iacopino AM. Periodontitis and diabetes interrelationships: role of inflammation. Ann Periodontol 6: 125-137, 2001.

3. Campus G, Salem A, Uzzau S, Baldoni E, Tonolo G. Diabetes and periodontal disease: a case-control study. J Periodontol 76: 418425, 2005.

4. Hung HC, Colditz G, Joshipura KJ. The association between tooth loss and the self-reported intake of selected CVD-related nutrients and foods among US women. Community Dent Oral Epidemiol 33: 167-173, 2005.

5. Takata Y, Ansai T, Awano S, et al. Relationship of physical fitness to chewing in an 80-year-old population. Oral Dis 10: 44-49, 2004.

6. Cutler CW, Shinedling EA, Nunn M, et al. Association between periodontitis and hyperlipidemia: cause or effect? J Periodontol 70: 1429-1434, 1999.

7. Wakai K, Kawamura T, Umemura O, et al. Associations of medical status and physical fitness with periodontal disease. J Clin Periodontol 26: 664-672, 1999.

8. Wu T, Trevisan M, Genco RJ, Falkner KL, Dorn JP, Sempos CT. Examination of the relation between periodontal health status and cardiovascular risk factors: serum total and high density lipoprotein cholesterol, C-reactive protein, and plasma fibrinogen. Am J Epidemiol 151: 273-282, 2000.

9. Katz J, Chaushu G, Sharabi Y. On the association between hypercholesterolemia, cardiovascular disease and severe periodontal disease. J Clin Periodontol 28: 865-868, 2001.

10. Buhlin K, Gustafsson A, Pockley AG, Frostegard J, Klinge B. Risk factors for cardiovascular disease in patients with periodontitis. Eur Heart J 24: 2099-2107, 2003.

11. Mattila KJ, Pussinen PJ, Paju S. Dental infections and cardiovascular diseases: a review. J Periodontol 76: 2085-2088, 2005.

12. Al-Zahrani MS, Kayal RA, Bissada NF. Periodontitis and cardiovascular disease: a review of shared risk factors and new findings supporting a causality hypothesis. Quintessence Int 37: 11-18, 2006.

13. Kshirsagar AV, Moss KL, Elter JR, Beck JD, Offenbacher S, Falk RJ. Periodontal disease is associated with renal insufficiency in the Atherosclerosis Risk In Communities (ARIC) study. Am J Kidney Dis 45: 650-657, 2005.

14. Thorstensson $\mathrm{H}$, Kuylenstierna J, Hugoson A. Medical status and complications in relation to periodontal disease experience in insulin-dependent diabetics. J Clin Periodontol 23: 194-202, 1996.

15. Furuichi Y, Shimotsu A, Ito H, et al. Associations of periodontal status with general health conditions and serum antibody titers for Porphyromonas gingivalis and Actinobacillus actinomycetemcomitans. J Periodontol 74: 1491-1497, 2003.

16. Taguchi A, Sanada M, Suei Y, et al. Tooth loss is associated with an increased risk of hypertension in postmenopausal women. Hypertension 43: 1297-1300, 2004.

17. Dalla Vecchia CF, Susin C, Rosing CK, Oppermann RV, Albandar JM. Overweight and obesity as risk indicators for periodontitis in adults. J Periodontol 76: 1721-1728, 2005.

18. Saito T, Shimazaki Y, Kiyohara Y, et al. Relationship between obesity, glucose tolerance, and periodontal disease in Japanese women: the Hisayama study. J Periodontal Res 40: 346-353, 2005.

19. Torrungruang $\mathrm{K}$, Tamsailom $\mathrm{S}$, Rojanasomsith $\mathrm{K}$, et al. Risk indicators of periodontal disease in older Thai adults. J Periodontol 76: 558-565, 2005.

20. Borges-Yanez SA, Irigoyen-Camacho ME, Maupome G. Risk factors and prevalence of periodontitis in community-dwelling elders in Mexico. J Clin Periodontol 33: 184-194, 2006.

21. Volzke H, Schwahn C, Dorr M, et al. Gender differences in the relation between number of teeth and systolic blood pressure. $\mathrm{J}$ Hypertens 24: 1257-1263, 2006.

22. Matsushima M. Japan Diabetes Society clinical practice guideline. Nippon Rinsho 60 suppl 9: 161-166, 2002 (in Japanese).

23. Kumasaka K. Test for microalbuminuria. Nippon Rinsho 62 suppl 11: 88-90, 2004 (in Japanese).

24. Van Dyke TE, Sheilesh D. Risk factors for periodontitis. J Int Acad Periodontol 7: 3-7, 2005.

25. Levey AS, Coresh J, Greene T, et al. Using standardized serum creatinine values in the modification of diet in renal disease study equation for estimating glomerular filtration rate. Ann Intern Med 145: 247-254, 2006.

26. SAS Institute Inc. Base SAS 9.1.3 Procedures Guide. 2nd ed. Cary NC, SAS Institute Inc., USA, 2006.

27. Chambless LE, Folsom AR, Davis V, et al. Risk factors for progression of common carotid atherosclerosis: the Atherosclerosis Risk in Communities Study, 1987-1998. Am J Epidemiol 155: 3847, 2002.

28. Goya K, Kitamura T, Inaba M, et al. Risk factors for asymptomatic atherosclerosis in Japanese type 2 diabetic patients without diabetic microvascular complications. Metabolism 52: 1302-1306, 2003.

29. Grant-Theule DA. Periodontal disease, diabetes, and immune response: a review of current concepts. J West Soc Periodontol Periodontal Abstr 44: 69-77, 1996.

30. Vilkuna-Rautiainen T, Pussinen PJ, Roivainen M, et al. Serum antibody response to periodontal pathogens and herpes simplex virus in relation to classic risk factors of cardiovascular disease. Int $\mathbf{J}$ Epidemiol 35: 1486-1494, 2006.

31. Dowse GK, Humphrey AR, Collins VR, et al. Prevalence and risk factors for diabetic retinopathy in the multiethnic population of Mauritius. Am J Epidemiol 147: 448-457, 1998.

32. Edwards MS, Hansen KJ, Craven TE, et al. Relationships between renovascular disease, blood pressure, and renal function in the elderly: a population-based study. Am J Kidney Dis 41: 990-996, 2003.

33. Haslam DW, James WP. Obesity. Lancet 366: 1197-1209, 2005.

34. UK Prospective Diabetes Study (UKPDS) Group. Intensive bloodglucose control with sulphonylureas or insulin compared with conventional treatment and risk of complications in patients with type 2 diabetes (UKPDS 33). Lancet 352: 837-853, 1998.

35. Sandberg GE, Sundberg HE, Fjellstrom CA, Wikblad KF. Type 2 diabetes and oral health: a comparison between diabetic and nondiabetic subjects. Diabetes Res Clin Pract 50: 27-34, 2000.

(C) 2007 The Japanese Society of Internal Medicine http://www.naika.or.jp/imindex.html 